\title{
Los hantavirus causantes \\ de la fiebre hemorrágica con síndrome renal y del síndrome pulmonar
}

\author{
Celso Ramos, QBP, D en C. ${ }^{(1)}$
}

\section{Ramos C. \\ Los hantavirus causantes de la fiebre hemorrágica con síndrome renal y del síndrome pulmonar. Salud Publica Mex 2008;50:334-340.}

\section{Resumen}

Este trabajo de revisión se enfoca en el análisis de la información básica sobre los hantavirus, agentes causales de dos enfermedades importantes en salud pública:la fiebre hemorrágica con síndrome renal (FHSR) y el síndrome pulmonar por hantavirus (SPH), dos zoonosis distribuidas en Asia/Europa y el continente americano, respectivamente. Los hantavirus se transmiten al hombre a través de la manipulación y contacto directos de roedores infectados o tejidos y secreciones (orina, heces y saliva). La FHSR y el SPH comparten algunas características clínicas, aunque las hemorragias y la afectación renal son propias de la FHSR,y los problemas respiratorios del SPH. Se aportan algunos datos sobre estudios realizados en México sobre hantavirus y se mencionan las condiciones ecológicas vinculadas con la distribución de los virus y sus reservorios naturales, así como algunas medidas para evitar o reducir el riesgo de infección.

Palabras clave: hantavirus; fiebre hemorrágica con síndrome renal; síndrome pulmonar por hantavirus; Sigmodontiniae; Peromyscus; México

\section{Ramos C.}

The hantaviruses causing hemorrhagic fever with renal syndrome and pulmonary syndrome. Salud Publica Mex 2008;50:334-340.

\section{Abstract}

The goal of this review is to provide basic information on hantaviruses as causative agents of Hemorrhagic Fever with Renal Syndrome (HFRS) and Hantavirus Pulmonary Syndrome (HPS), two zoonotic diseases widely distributed in Asia/Europe, and the American continent, respectively. Hantaviruses are rodent-borne and transmitted to humans by direct contact with infected rodents or their secretions (urine, feces and saliva). Both, HFRS and HPS share some clinical aspects, however, hemorrhage and renal failure are the hallmark of HFRS, while respiratory problems are distinctive signs and symptoms of patients with HPS. Studies on hantavirus infection in rodents from Mexico are included, some recomendations to prevent or avoid contact with rodents are mentioned, and some determinant ecologic factors of hantaviruses distribution and their natural rodents, are also included.

Key words: hantavirus; hemorrhagic fever with renal syndrome; hantavirus pulmonary syndrome; Sigmodontiniae; Peromyscus; Mexico

(I) Departamento de Arbovirus, Centro de Investigaciones sobre Enfermedades Infecciosas, Instituto Nacional de Salud Pública. Cuernavaca, Morelos, México.

Fecha de recibido: 12 de noviembre de 2007 - Fecha de aceptado: 7 de mayo de 2008

Solicitud de sobretiros: Dr. Celso Ramos. Centro de Investigaciones sobre Enfermedades Infecciosas, INSP.Av. Universidad 655, Col. Santa María Ahuacatitlán. 62508 Cuernavaca, Morelos, México.

Correo electrónico: cramos@insp.mx 
L os hantavirus (familia Bunyaviridae, género hantavirus) infectan a una diversidad de roedores y son los agentes causales de la fiebre hemorrágica con síndrome renal (FHSR), que se distribuye sobre todo en varios países de Asia y Europa, y el síndrome pulmonar por hantavirus (SPH), con prevalencia en el continente americano. ${ }^{1-3}$ Los hantavirus se propagan entre los roedores y el ser humano a través del contacto directo con secreciones (orina, heces y saliva) de animales infectados o mediante aerosoles que contienen al virus; en los roedores tiene lugar una infección crónica persistente que casi nunca causa enfermedad o muerte; este fenómeno se debe a un proceso de coevolución entre los virus y sus hospedadores reservorios que ha ocurrido a lo largo de miles de años y que explica la relación específica de especie de los virus con sus reservorios naturales. ${ }^{1,4-7}$

A pesar de que existe una clara distribución geográfica de los casos de FHSR y de SPH, en fecha reciente se han descrito algunos casos importados del SPH a zonas no endémicas. ${ }^{89}$ El diagnóstico de ambas enfermedades se establece mediante técnicas serológicas y de biología molecular y para el aislamiento y caracterización de los virus se requiere un laboratorio de bioseguridad de nivel 3, ya que los hantavirus se consideran agentes infecciosos de alto riesgo ${ }^{10-12}$ e incluso están incluidos en el inventario de agentes patógenos de uso potencial para el bioterrorismo. ${ }^{13}$ Estos microorganismos tienen un genoma de tres segmentos de RNA de polaridad negativa; ${ }^{14}$ la diversidad genética vinculada con estos virus se debe en parte a procesos de reasociación de los segmentos de RNA, que puede explicar la diversidad de los virus conocidos hasta la fecha. ${ }^{4-7,15-17}$

El incremento de los casos del SPH en el continente americano se debe en parte a la amplia distribución de los roedores reservorios, la gran diversidad del virus, la población susceptible, los riesgos de exposición y el reconocimiento y notificación de la enfermedad por el personal médico. ${ }^{16} \mathrm{El}$ objetivo de esta revisión es suministrar información básica al personal médico, a las autoridades y trabajadores de la salud y al personal ocupacionalmente expuesto a la enfermedad acerca de la prevención y el control para reducir los riesgos de exposición. Esta revisión incluye información publicada desde la detección de los primeros casos en Estados Unidos de América en 1993 hasta los hallazgos recientes de infección en roedores y seres humanos notificados en Latinoamérica, incluido México.

\section{Fiebre hemorrágica con síndrome renal (FHSR)}

Los primeros registros de FHSR se reconocieron a principios del decenio de 1950, con más de 3200 casos informados entre 1951 y 1954 entre las tropas de las Naciones Unidas establecidas en Corea. ${ }^{1,4}$ La FHSR es causada por un grupo de virus, de los cuales el hantaan es el prototipo y los ratones Apodemus agrarius son su reservorio natural (letalidad de 5 a $15 \%$ ). ${ }^{1,4}$ Hoy en día se comunican en China más de 100000 casos al año y en varios países de Europa se conocen otros virus (p. ej., Puumala, Dobrava, Seoul, etc.) que causan enfermedades menos graves que la FHSR. La distribución geográfica de los hantavirus se ha extendido a varios países de África. ${ }^{4}$

\section{Cuadro clínico}

La FHSR se presenta en etapas progresivas que se caracterizan por fiebre, estado de choque o hipotensión, oliguria, poliuria y convalecencia. ${ }^{18-20}$ La aparición de los síntomas casi siempre es repentina con intenso dolor de cabeza, dolor de espalda, fiebre y escalofrío; la hemorragia, si ocurre, se manifiesta durante la fase febril y la albuminuria se presenta en los casos graves. Después de la etapa febril se desarrolla la hipotensión, que puede durar algunas horas o días, y durante la cual puede haber náusea y vómito. Alrededor de $30 \%$ de las muertes ocurre en esta etapa debido a la permeabilidad vascular y choque agudo. Los pacientes que sobreviven y progresan a la fase diurética muestran mejoría de la función renal, pero existe el riesgo de morir por complicaciones pulmonares o choque. En las fases tempranas de la enfermedad, los pacientes pueden tener trombocitopenia, niveles alterados de los productos de la degradación de la fibrina sérica y fibrinógeno, alteraciones del tiempo parcial de tromboplastina, protrombina y tiempos de coagulación; en estudios experimentales en roedores y casos humanos se ha demostrado una extensa infección de células del endotelio vascular y en biopsias renales se ha detectado antígeno en células tubulares; los riñones son los órganos más afectados y el origen de la insuficiencia renal es tal vez una combinación del choque sistémico y condiciones de la circulación renal local. A diferencia de la mayoría de las fiebres hemorrágicas, la respuesta inmunitaria (p. ej., anticuerpos neutralizantes y linfocitos CD8+ activados) en pacientes con FHSR se presenta en las etapas tempranas de la infección; hay evidencias que sugieren que la reacción inmunitaria puede ser un mecanismo inductor de la patogenia de la FHSR. ${ }^{14}$

El diagnóstico de este trastorno se determina por la detección de anticuerpos, el RNA del virus (RT-PCR) e inmunohistoquímica; ${ }^{20}$ el aislamiento del virus se puede realizar en cultivo de células o en animales de experimentación en laboratorios de bioseguridad de nivel $3 .^{21,22} \mathrm{No}$ existe un tratamiento específico, aunque algunos autores 
han demostrado la utilidad de la ribavirina. ${ }^{23}$ Tampoco existen vacunas efectivas para esta enfermedad, si bien hay información sobre el desarrollo y ensayo de algunas vacunas, el uso de la biotecnología moderna coadyuvará al desarrollo de esta medida de prevención. ${ }^{24-26}$

\section{Síndrome pulmonar por hantavirus (SPH)}

Hasta hace algunos años se desconocía la presencia del hantavirus en el continente americano; empero, en 1993 se registró un brote de una enfermedad respiratoria que se denominó síndrome pulmonar por hantavirus (SPH) en la región Four Corners, ubicada en una zona compartida por Utah, Nuevo México, Arizona y Colorado. 2,27 El SPH tiene una elevada tasa de mortalidad (cercana a 40\%) y las autoridades de salud (Centers for Disease Control and Prevention, $\mathrm{CDC}$ ) reconocieron un nuevo hantavirus (virus sin nombre, VSN) como el agente causal de la enfermedad. ${ }^{1,27-30}$ Los estudios epidemiológicos demostraron la presencia del virus en secreciones, heces y orina de ratones silvestres recolectados en la región, en particular del género Peromyscus maniculatus. ${ }^{27}$ Estudios retrospectivos realizados en diversas regiones de Norteamérica sugieren casos similares desde finales de la década de 1950 y por lo tanto es factible que este virus sea endémico de la región. . $^{1,27-30}$ Investigaciones posteriores demostraron la presencia de roedores infectados y casos de SPH en otras regiones de Estados Unidos de América y Canadá., 27-33 Sin embargo, otros hantavirus (p. ej., El Moro Canyon, Tula, Isla Vista y Prospect Hill) no causan la enfermedad. ${ }^{1,22}$

\section{Cuadro clínico}

Las manifestaciones tempranas de la enfermedad son fiebre, mialgia, malestar general, disnea y otros síntomas respiratorios, ${ }^{34}$ aunque es necesario establecer la diferencia con el síndrome de dificultad respiratoria del adulto, que ocurre en individuos con enfermedad pulmonar crónica. Los pacientes presentan hemoconcentración (extravasación del plasma sobre todo a los pulmones), hipoxemia, taquicardia e hipotensión; en esta fase de la enfermedad se pueden detectar anticuerpos y linfocitos CD8+ activados (linfoblastos) en la sangre periférica. La afectación pulmonar progresa con rapidez y alrededor de $40 \%$ de los sujetos puede fallecer dentro de las 48 horas tras la admisión al hospital. La fisiopatología pulmonar más evidente es el edema pulmonar. Las causas del incremento de la permeabilidad vascular no se conocen con precisión, a pesar de que el daño epitelial es escaso. ${ }^{14}$

La fiebre, la trombocitopenia y la leucocitosis son comunes a la FHSR y el SPH; empero, el dato clínico que distingue a los pacientes con SPH es la fragilidad capilar que se localiza de manera exclusiva en los pulmones; los riñones rara vez se afectan, como sucede en los enfermos con FHSR. ${ }^{14}$

Se ha demostrado que la integrina $\alpha v \beta 3$ es el receptor para los hantavirus y se halla presente en la superficie de células endoteliales y plaquetas, si bien otras moléculas de adhesión (p. ej., ICAM-1, VECAM y PECAM) se expresan durante la infección ${ }^{35}$ y pueden coadyuvar en la fisiopatología de la enfermedad; algunos sujetos con cuadro grave de SPH tienen una mayor expresión del alelo HLA-B35y puede considerarse como un marcador de riesgo.

La transmisión de los hantavirus de persona a persona es infrecuente en Estados Unidos de América; así lo demuestran estudios realizados en personal de salud que atiende a pacientes con SPH. Sin embargo, en Argentina se ha demostrado la transmisión del hantavirus (virus Andes) de persona a persona; ${ }^{36}$ Padula y colaboradores demostraron esta vía de transmisión en personal médico que atendió a individuos o en familiares que tuvieron contacto cercano con los pacientes de SPH. ${ }^{36}$ No obstante, en otros países esta vía de transmisión no se conoce o no se ha demostrado, ya que la infección se adquiere casi siempre por la exposición a secreciones, orina o heces de roedores infectados.

El diagnóstico de laboratorio se determina mediante la detección de anticuerpos $\operatorname{IgM}$ o incremento de la IgG; la RT-PCR y la inmunohistoquímica pueden ser herramientas útiles para confirmar la infección. 10,11,21,37

Algunos otros datos de laboratorio en personas con SPH son la disminución de la albúmina, incremento del hematócrito, fuga capilar, aumento de linfocitos atípicos, proteinuria, elevación de las transaminasas, amilasa y creatinina, alteraciones de la coagulación, disminución del fibrinógeno, incremento de productos de la fibrina y trombocitopenia. La acidosis metabólica y los niveles elevados del lactato sérico representan un mal pronóstico . Algunos mediadores de la respuesta inmunitaria como el TNF- $\alpha$ pueden ser causantes del incremento de la permeabilidad vascular pulmonar y la hemoconcentración. No existe tratamiento específico para el SPH y en pacientes graves se debe vigilar el volumen y la función cardiaca (mortalidad de 40\%). El diagnóstico diferencial incluye la búsqueda de infección por diversos agentes patógenos (p. ej., leptospira, enfermedad de los legionarios, histoplasmosis, etc.). ${ }^{1,10,14,16,22}$

Luego de los casos notificados en la región Four Corners, se reconocieron otros en Nueva York, Florida y Luisiana. En 1997, Rhodes y colaboradore ${ }^{31}$ comunicaron los dos primeros casos fatales del SPH en el estado de Pensilvania. 
El SPH es más común en países de Sudamérica (p. ej., Argentina, Chile, Uruguay, Paraguay, Brasil y Bolivia) ${ }^{36,38-44}$ que en Estados Unidos. Aunque esta enfermedad es más común en áreas rurales, también puede surgir en zonas suburbanas donde abundan los roedores silvestres. ${ }^{1,4,16,22}$ Además del VSN, otros hantavirus se han vinculado con el SPH, entre ellos los virus Black Creek, Bayou y New York. En Sudamérica diversos hantavirus se han relacionado con casos de SPH. $1,16,22,38-44$

Es importante que el personal médico que atiende a pacientes con FHSR y SPH utilice el material de protección adecuado indicado, lo cual vale también para investigadores y trabajadores de la salud (biólogos, veterinarios, mastozoólogos, etc.) que están en contacto con roedores silvestres, tejidos o secreciones. ${ }^{45-47}$ Para las personas que viven en zonas endémicas o aquellas que realizan actividades laborales, deportivas o turísticas, se recomienda evitar el contacto con roedores o sus secreciones a fin de disminuir el riesgo de infección, para lo cual se recomiendan algunas actividades como no exponerse al polvo o aerosoles en casas abandonadas donde abundan los roedores silvestres, evitar la entrada de roedores a los hogares, utilizar guantes y cubrebocas cuando se manipula a animales muertos, usar desinfectantes caseros en áreas contaminadas con heces de roedores y no tener alimento para mascotas cerca del hogar. ${ }^{1,10,16,22}$

\section{Estudios sobre hantavirus en México}

México tiene una gran diversidad de roedores que pueden ser reservorios potenciales de los hantavirus, ya que se ubican en una región geográfica comprendida entre Norteamérica y América Central y Sudamérica. ${ }^{48}$ En consecuencia, de más de 120 especies conocidas de sigmodontinos en México, alrededor de 80 pertenecen a cinco géneros (Peromyscus, Reithrodontomys, Oligoryzomys, Oryzomys y Sigmodon) que son reservorios para los hantavirus. A pesar de la ubicación geográfica del país, la abundancia de roedores y población humana susceptible a la infección por hantavirus, no hay mucha información o estudios sistemáticos sobre la búsqueda de infección en roedores o casos de SPH. En el año 2001 Mantooth y colaboradores notificaron un hantavirus (El Moro Canyon) en México, que se identificó en Reithrodondomys megalotis. ${ }^{32}$ En un estudio que se realizó en 2003 en Yucatán en personas asintomáticas, se registraron cuatro individuos con anticuerpos contra hantavirus, pero no se hallaron anticuerpos en roedores colectados en la región. ${ }^{49}$ Otros estudios serológicos o moleculares efectuados en muestras de diferentes roedores de Oaxaca y Zacatecas han mostrado evidencia de infección por hantavirus en R. megalotis, R. sumichrasti, P. maniculatus, $P$. melanosis y P. hylocete. ${ }^{50}$ En un estudio serológico que publicaron en el año 2007 Castro-Arellano y colaboradores se muestra evidencia de infección por hantavirus en ratones Peromyscus levipes colectados en Tamaulipas, México. ${ }^{51}$

En fecha reciente, Chu y colaboradores ${ }^{52}$ realizaron la caracterización genética y filogenética de un nuevo hantavirus, al que denominaron Playa Oro (POV), que se reconoció en roedores Oryzomys couesi capturados en 2004 en el estado de Colima; Milazzo y colegas identificaron en este mismo roedor al hantavirus denominado Catacamas en Honduras en 2006. ${ }^{53}$ En el estudio de Chu y colaboradores, ${ }^{52}$ los ratones Sigmodon mascotensis tuvieron títulos altos de anticuerpos, seguidos por Oryzomys couesi y Baiomys musculus; empero, O. couesi tuvo los valores más altos y RNA viral, por lo que se puede considerar como el reservorio principal del POV.

De igual modo, un grupo de investigadores del INSP, la Universidad de Hokkaido (Sapporo, Japón), la UNAM y la UAEM (Morelos) realizó un estudio en roedores silvestres para la búsqueda de infección por hantavirus; se hicieron dos colectas en los años 2006 y 2007 en algunas regiones de los estados de Guerrero y Morelos. Los estudios serológicos (ELISA y western blot) del primer estudio muestran una seroprevalencia de 13.2\%, similar a la observada en Estados Unidos y otros países endémicos de Sudamérica, así como la detección del genoma del virus mediante RT-PCR en una variedad de roedores (Habromys simulatus, Habromys spp., Peromyscus aztecas, P. beatae, Reithrodontomys fulvescens, R. megalotis y R. sumichrasti); el análisis de la secuencia de nucleótidos del segmento $S$ de los virus analizados (denominados BEA, AZT y FUL) y su comparación filogenética con otros hantavirus los ubica en posiciones diferentes a las descritas para los virus prototipo VSN y Andes; además, los hantavirus identificados en este estudio se relacionan con roedores silvestres endémicos de México (manuscrito en preparación).

\section{Aspectos ecológicos de los hantavirus y sus reservorios}

Los hantavirus han coevolucionado con sus reservorios naturales (roedores), en los que producen una infección persistente (coespeciación). ${ }^{6-7}$ Diversos estudios epidemiológicos indican una vinculación específica de especie entre los hantavirus y los roedores; de este modo se puede explicar la presencia de la FHSR en Asia y Europa, donde abundan los ratones Apodemus agrarius, $A$. flavicollis y Clethrionomys glaereolus, a diferencia del SPH que se relaciona en especial con ratones de la especie Peromyscus maniculatus en Norteamérica, 
Canadá y países de Latinoamérica. ${ }^{1,54}$ Estudios recientes del genoma del VSN señalan que no se introdujo de Asia o Europa, sino más bien que ha tenido una historia evolutiva de muchos años en el continente americano. De los 28 hantavirus descritos hasta la fecha, por lo menos 16 son patógenos para el hombre. La abundancia de alimentos y las condiciones ambientales favorables para la proliferación de los roedores pueden posibilitar la invasión del ambiente doméstico o peridoméstico por roedores infectados, lo cual eleva el riesgo de infección en seres humanos. ${ }^{16,22,54}$

A pesar de que no es común la presencia de casos de FHSR en el continente americano, es importante que el personal médico conozca los principales signos y síntomas de la enfermedad, ya que pueden ocurrir casos importados de personas que visitan zonas endémicas de Europa y Asia. ${ }^{8,9}$ En la actualidad hay pocos casos nativos de FHSR en América y aquellos que se han notificado se deben al virus Seoul, cuyo reservorio es la rata común (Rattus rattus) que posee una distribución universal y es causante de la diseminación del virus en áreas urbanas. ${ }^{1,4}$

Aunque los roedores son los principales reservorios del virus sin nombre en América, se han informado hallazgos de anticuerpos contra el VSN en otros animales domésticos y silvestres (p. ej., gatos, perros, cerdos, liebres, musarañas, ratas, aves, primates no humanos y venados). ${ }^{4}$

En un estudio de Sánchez-Cordero y colaboradores ${ }^{48}$ se determinó la distribución geográfica de los roedores del VSN y su nexo con casos de SPH mediante un modelo extraído de la información disponible en Estados Unidos de América; este estudio estuvo enfocado en identificar posibles regiones de riesgo en México. Los casos de SPH detectados en seres humanos en Estados Unidos de América coincidieron en forma significativamente mayor con la distribución de los reservorios. En México, los roedores reservorios del SNV cubren extensas zonas geográficas que se consideran regiones de riesgo para la transmisión del virus. La similitud de las condiciones biológicas, ecológicas y sociales de amplias regiones de México y Estados Unidos hizo posible ubicar y detectar casos de infección en roedores y personas mediante el uso de la herramienta de trabajo descrita en esta investigación.

Sin duda, la circulación de hantavirus en México, junto con las condiciones ambientales que permiten la proliferación de los roedores reservorios, así como la población susceptible, ubica al país en un alto riesgo para la aparición de casos del SPH, por lo que es importante difundir entre la comunidad médica, investigadores, autoridades de salud y personas en riesgo información básica que coadyuve a fortalecer las acciones de vigi- lancia, prevención y control de este padecimiento poco conocido en México. La información revisada aporta no sólo datos históricos de ambos padecimientos causados por los hantavirus, sino también datos recientes sobre el avance en el conocimiento de este tema. Por último, es importante fortalecer la investigación en roedores y seres humanos, así como apoyar el establecimiento de infraestructura de laboratorio (bioseguridad de niveles 3 y 4), la formación de recursos humanos y el entrenamiento de personal.

\section{Agradecimientos}

Agradezco la colaboración del Biól. Exp. Ángel Ramírez Castellanos, estudiante de la Maestría en Ciencias con Área de Concentración en Enfermedades Infecciosas del CISEI/INSP de quien fueron valiosos su apoyo para la búsqueda de información y sus comentarios a este trabajo.

\section{Referencias}

I. Schmaljohn C, Hjelle B. Hantaviruses: a global disease problem. Emerg Infect Dis 1997;3:95-104.

2. Nichol ST, Spiropoulou CF, Morzunov S, Rollin PE, Ksiazek TG, et al. Genetic identification of a hantavirus associated with an outbreak of acute respiratory illness. Science 1993;262:914-917.

3. Padula P, Martinez VP, Bellomo C, Maidana S, San Juan J, Tagliaferri P, et al. Pathogenic hantaviruses, Northeastern Argentina and Eastern Paraguay. Emerg Infect Dis 2007;13:1211-1214.

4. Zeier M, Handeremann M, Bahr U, Rensch B, Muller S, Kehm, R, et al. New ecological aspects of hantavirus infection: a change of a paradigm and a challenge of prevention- a review.Virus Genes 2005;30:157-180.

5. Elliot RM, Schmaljohn CS, Collett MS. Bunyaviridae genome structure and gene expression. In: Kolakofsky D, ed. Current topics in microbiology and immunology.Vienna: Springer-Verlag, 1991:91-141.

6. Plyusnin A, Morzunov SP.Virus evolution and genetic diversity of hantaviruses and their hosts. Cur Top Microbiol Immunol 200 I;256:47-75. 7. Yates TL, Mills JN, Parmenter CA. The ecology and evolutionary history of an emergent disease: hantavirus pulmonary syndrome. Bioscience 2002;52:989-998.

8. Murgue B, Domart Y, Coudrier D, Rollin PE, Darchis JP, Merrien D. First reported case of imported hantavirus pulmonary syndrome in Europe. Emerg Infect Dis 2002;8:106-107.

9. Reynolds S, Galanis E, Krajden M, Morshed M, Bowering D,Abelson W, et al. Imported fatal hantavirus pulmonary syndrome. Emerg Infect Dis 2007; 13:1424-1425.

10. Centers for Disease Control and Prevention. Laboratory management of agents associated with hantavirus pulmonary syndrome: interim biosafety guidelines. Morb Mortal Wkly Rept 1994;7:I-2.

II. Song JW, Back LJ, Nagle JW, Schlitter D, Yanagihara R. Genetic and phylogenetic analyses of hantaviral sequences amplified from archival tissues of deer mice (Peromyscus maniculatus nubiterrae) captured in the eastern United States. Arch Virol 1996; 141:959-967.

12. Botten J, Mirowsky K, Kusewitt D, Bharadwaj M, Yee J, Ricci R, et al. Experimental infection model for Sin Nombre hantavirus in the deer 
mouse (Peromyscus maniculatus). Proc Natl Acad Sci USA 2000;97:1057810583.

13. Centers for Disease Control and Prevention. [Consultado en mayo de 2006]. Disponible en: http://www.cdc.gov/hantavirus.

14. Maes P, Clement J, Gavrilovskaya I,Van Ranst M. Hantaviruses: immunology, treatment, and prevention. Review.Viral Immunol 2004; 17:48I-497.

15. Moreli ML, Sousa RL, Figueiredo LT. Detection of Brazilian hantavirus by reverse transcription polymerase chain reaction amplification of $\mathrm{N}$ gene in patients with hantavirus cardiopulmonary syndrome. Mem Inst Oswaldo Cruz 2004;99:633-638.

16. Hantavirus in the Americas: guidelines for diagnosis, treatment, and control. Cuaderno Técnico No. 47. Washington, DC: Panamerican Health Organization, 1999.

17. Hjelle B, Anderson B, Torres-Martinez N, Song W, Gannon WL, Yates TL. Prevalence and geographic genetic variation of hantaviruses of New World harvest mice (Reitrodontomys): identification of a divergent genotype from a Costa Rican Reithrodontomys mexicanus. Virology 1995;207:452-459. 18. Chen LB, Yang WS. Abnormalities of T cell immunoregulation in hemorrhagic fever with renal syndrome.J Infect Dis 1990;161:1016-1019. 19. Yanigahara R, Silverman DJ. Experimental infection of human vascular endothelial cells by pathogenic and nonpathogenic hantaviruses. Arch Virol 1990; | | I:28I-286.

20. Peters C], Simpson GL, Levy H. Spectrum of hantavirus infection: hemorrhagic fever with renal syndrome and hantavirus pulmonary syndrome. Annu Rev Med 1999;50:531-545.

2I. Hjelle B, Jenison S, Torrez-Martinez N. Rapid and specific detection of $\mathrm{Sin}$ Nombre virus antibodies in patients with hantavirus pulmonary syndrome by a strip immunoblot assay suitable for field diagnosis. J Clin Microbiol 1997;35:600-608.

22. Centers for Disease Control and Prevention. All about hantavirus. Atlanta, Ga: National Center for Infectious Diseases. Special Pathogens Branch, 2006.

23. Huggins JW, Hsiang CM, Cosgriff TM. Prospective, double-blind, concurrent, placebo controlled clinical trial of intravenous ribavirin therapy of hemorrhagic fever with renal syndrome.J Infect Dis 199|; |64:III I-I I 27.

24. Cho HW, Howard CR. Antibody responses in humans to an inactivated hantavirus vaccine (Hantavax).Vaccine 1999;17:2569-2575.

25. Park K, Kim CS, Moon KT. Protective effectiveness of hantavirus vaccine. Emerg Infect Dis 2004;10:2218-2220.

26. Cho HW, Howard CR, Lee HW. Review of an inactivated vaccine against hantaviruses. Intervirology 2002;45:328-333.

27. Childs JE, Ksiazek TG, Spiropoulou CF. Serologic and genetic identification of Peromyscus maniculatus as the primary rodent reservoir for a new hantavirus in the southwestern United States.J Infect Dis 1994; 169:127I-1280.

28. Duchin JS, Koster F, Peters CJ. Hantavirus pulmonary syndrome: a clinical description of 17 patients with a newly recognized disease. N Engl J Med 1994:330:949-955.

29. Nolte KB, Feddersen RM, Foucar K. Hantavirus pulmonary syndrome in the United States: a pathological description of a disease caused by a new agent. Hum Pathol 1995;26:1 I0-120.

30. Monroe MC, Morzunov SP, Johnson AM. Genetic diversity and distribution of Peromyscus-borne hantaviruses in North America. Emerg Infect Dis 1999;5:75-86.

3I. Rhodes LV, Huang C, Sanchez AJ, Nichol ST, Zaki SR, Ksiazek TG, et al. Hantavirus pulmonary syndrome associated with Monongahela virus, Pennsylvania. Emerg Infect Dis 2000;6:616-621.

32. Mantooth SJ, Milazzo ML, Bradley RD, Hice CL, Ceballos G, Tesh RB, et al. Geographical distribution of rodent-associated hantaviruses in Texas.J Vector Ecol 200I;26:7-14.
33. Sinclair JR, Carroll DS, Montgomery JM, Pavlin M, McCombs K, Mills JN, et al. Two cases of hantavirus pulmonary syndrome in Randolph County, West Virginia: a coincidence of time and place? Am J Trop Med Hyg 2007;76:438-442.

34. Centers for Disease Control and Prevention. Case Information: hantavirus pulmonary syndrome case count and descriptive statistics. Available at http://www.cdc.gov/ncidod/diseases/hanta/noframes/caseinfo. htm. [Access on: february 27, 2006.]

35. Gavrilovskaya IN, Shapley M, Shaw R, Ginsberg MH, Mackow ER. 3 integrins mediate the cellular entry of hantaviruses that cause respiratory failure. Proc Natl Acad Sci USA 1998;95:7074-7079.

36. Padula PJ, Edelstein A, Miguel SDL, Lopez NM, Rossi CM, Rabinovich RD. Hantavirus pulmonary syndrome outbreak in Argentina: molecular evidence for person-to- person transmission of Andes virus. Virology 1998;241:323-330.

37. Elgh F, Lundkvist A,Alexeyev OA. Serological diagnosis of hantavirus infections by an enzyme-linked immunosorbent assay based on detection of immunoglobulin $\mathrm{G}$ and $\mathrm{M}$ responses to recombinant nucleocapsid proteins of five viral serotypes. J Clin Microbiol I997;35: I I22-I I30. 38. Johnson AM, Bowen MD, Ksiazek TG, Williams RJ, Bryan RT, Mills JN, et al. Laguna Negra virus associated with HPS in western Paraguay and Bolivia.Virology 1997;238:1 I5-127.

39. Lopez N, Padula P, Rossi C, Miguel S, Edelstein A, Ramirez E, et al. Genetic characterization and phylogeny of Andes virus and variants from Argentina and Chile.Virus Res 1997;50:77-84.

40. Levis S, Morzunov S, Rowe JE, Enria DA, Pini N, Calderon G. Genetic diversity and epidemiology of hantavirus in Argentina.J Infect Dis 1998; 177:529-538.

4I. Chu YK, Owen RD, Gonzalez LM, Jonsson CB. The complex ecology of hantavirus in Paraguay. Am J Trop Med Hyg 2003;69:263-268.

42. Rosa EST, Mills NJ, Padula PJ. Newly recognized hantavirus associated with hantavirus pulmonary syndrome in northern Brazil: partial genetic characterization of viruses and serologic implication of likely reservoirs. Vector Borne Zoonotic Dis 2005;5: I I- 19.

43. Carroll DS, Mills JN, Montgomery JM, Bausch DG, Blair PJ, Burans JP, et al. Hantavirus pulmonary syndrome in Central Bolivia: relationships between reservoir hosts, habitats, and viral genotypes. Am J Trop Med Hyg 2005;72:42-46.

44. Raboni SM, Rubio G, De Borba I, Zeferino A, Skraba I, Goldenberg S. Clinical survey of hantavirus in southern Brazil and the development of specific molecular diagnosis tools. Am J Trop Med Hyg 2005;72:800-804. 45. Mills JN, Yates TL, Childs JE, Parmenter RR, Ksiazek TG, Rollin PE, et al. Guidelines for working with rodents potentially infected with hantavirus.J Mammology 1995;76:716-722.

46. Mills JN, Childs JE, Ksiazek TG, Peters CJ,Velleca WN. Methods for trapping and sampling small mammals for virologic testing. US Department of Health \& Human Services. Public Helath Service. Atlanta, Ga: Centers for Disease Control and Prevention, 1995.

47. Kelt DA, Van Vuren DH, Hafner MS, Danielson BJ, Kelly MJ.Threat of hantavirus pulmonary syndrome to field biologists working with small mammals. Emerg Infect Dis 2007; 13:1285- 287.

48. Sánchez-Cordero V, Peterson AT, Martínez-Meyer E, Flores R.

Distribución de roedores reservorios del virus causante del síndrome pulmonar por hantavirus y regiones de posible riesgo de México.Acta Zool Mex 2005;21:79-91.

49.Vado-Solís I, Pérez-Osorio C, Lara-Lara H, Ruiz-Piña HA, CárdenasMarrufo M, Milazzo ML, et al. Evidencia serológica de infección por hantavirus en población humana del estado de Yucatán, México. Rev Biomed 2003;| 4:22I-225.

50. Suzan G, Ceballos G, Mills J, Ksiazek TG, Yates T. Serologic evidence of hantavirus infection in Sigmodontine rodents in Mexico.JWild Dis 2001;37:391-393. 
5I. Castro-Arellano I, Suzan G, Flores-Leon R, Morales-jimenez R, Lacher TE. First report of hantavirus occurrence in the nimble-footed mouse Peromyscus levipes, in northern Mexico. Peromyscus Newsletter 2007; $42: 36$.

52. Chu YK, Owen R, Sanchez-Hernandez C, Romero-Almaraz ML, Jonsson CB. Genetic characterization and phylogeny of a hantavirus from Western México.Virus Res 2007;|3|:|80-|88.

53. Milazzo ML, Cajimat MN, Hanson JD, Bradley RD, Quintana M, Sherman $C$, et al. Catacamas virus, a hantaviral species naturally associated with Oryzomys couesi in Honduras. Am JTrop Med Hyg 2006;75: 1003-1010. 54. Dragoo JW, Lackey JA, Moore KE, Lessa EP, Cook JA, Yates TL.

Phylogeography of the deer mouse (Peromyscus maniculatus) provides a predictive framework for research on hantavirus. J Gen Virol 2006;87:1997-2003.

55. Armstrong L, Zaki S, Goldoft M,Todd R, Khan A, Khabbaz R. Hantavirus pulmonary syndrome associated with entering or cleaning rarely used rodent-infested structures.J Infect Dis 1995; I72: I 66.

56. Kilpatrick ED, Terajima M, Koster Ft, Catalina MD, Cruz J, Ennis FA. Role of specific CD8+T cells in the severity of a fulminant zoonotic viral hemorrhagic fever, hantavirus pulmonary syndrome. J Immunol 2004; I72:3297-3304. 